Article

\title{
Cholesteryl-Modification of a Glucomannan from Bletilla striata and Its Hydrogel Properties
}

\author{
Mengshan Zhang ${ }^{1}$, Lin Sun ${ }^{1}$, Wencui Zhao ${ }^{2}$, Xiaoxia Peng ${ }^{1}$, Fuqiang Liu ${ }^{2}$, Yanping Wang ${ }^{2}$, \\ Yajing Bi ${ }^{2}$, Hengbi Zhang ${ }^{2}$ and Yifa Zhou ${ }^{1, *}$ \\ 1 School of Life Sciences, Northeast Normal University, Changchun 130024, China; \\ E-Mails: zhangms253@nenu.edu.cn (M.Z.); sun1925@nenu.edu.cn (L.S.); \\ pengxx039@nenu.edu.cn (X.P.) \\ 2 Pharmacy Department, Chinese People's Liberation Army 208 Hospital, Changchun 130062, \\ China; E-Mails: zhaowencui208@126.com (W.Z.); cclfq@126.com (F.L.); \\ aswangyanping@163.com (Y.W.); asbiyajing@163.com (Y.B.); zqi8229@126.com (H.Z.) \\ * Author to whom correspondence should be addressed; E-Mail: zhouyf383@nenu.edu.cn; \\ Tel./Fax: +86-431-8509-8212.
}

Received: 25 May 2014; in revised form: 21 June 2014 / Accepted: 24 June 2014 /

Published: 30 June 2014

\begin{abstract}
A glucomannan-type polysaccharide, named BSP, was obtained from the tubers of Bletilla striata by ultrasonic-assisted extraction, ethanol precipitation, deproteination and gel-permeation chromatography. HPLC analysis revealed that BSP contained mannose and glucose in the molar ratio of 3.5:1. Its molecular weight $(\mathrm{Mw})$ was estimated to be $20 \mathrm{kDa}$. Methylation analysis, FT-IR and NMR analyses indicated that BSP consisted of $(1 \rightarrow 4)$-linked $\beta$-D-glucopyranosyl residues and $(1 \rightarrow 4)$-linked $\beta$-D-mannopyranosyl residues. Cholesteryl succinate was linked to BSP to make it more amphiphilic and the degree of substitution of cholesteryl succinate-BSP was $3.2 \%$. The critical micelle concentration of modified BSP was $0.001 \mathrm{mg} / \mathrm{mL}$, suggesting it could self-assemble into nanoparticles in aqueous solution.
\end{abstract}

Keywords: cholesteryl-modification; glucomannan; Bletilla striata; hydrogel property 


\section{Introduction}

Bletilla striata (Thunb.) Reichb. $f$. is a perennial herbaceous plant, used as a traditional medicine for thousands of years in East Asian countries [1]. Bletilla striata polysaccharide (BSP) gel is a natural hydrogel used for preparing biological adhesives [2]. BSP microspheres have been prepared for directed targeting, drug delivery, controlled release and for their anti-tumor activities [3]. Glucomannan with a backbone of $(1 \rightarrow 4)$-linked $\beta$-D-mannose and glucose in general is the dominant neutral polysaccharide in Bletilla striata [4,5]. A recent study reported a new glucomannan obtained by hot water extraction from Bletilla striata and subsequent purification by ion-exchange chromatography that was mainly composed of $(1 \rightarrow 2)$-linked $\alpha$-D-mannopyranose and $(1 \rightarrow 4)$-linked $\beta$-D-glucopyranose residues [6]. It is also reported that the tubers of Bletilla striata contained abundant glucomannan, responsible for its bioactivities [7]. Although polysaccharides from Bletilla striata possess various pharmacological functions, few studies have reported the utilization of these polysaccharides as drug carriers. Bletilla striata polysaccharides are water-soluble polysaccharides which lack a hydrophobic domain structure, so they are not good enough to coat fat-soluble drugs and the drug delivery yield is therefore low, making it necessary to find appropriate chemical means to modify them in order to improve their hydrogel strength and ability to coat drugs. Many polysaccharides, for instance pullulan [8] and carboxymethyl cellulose [9], had been successfully modified with cholesterol. This paper reports a cholesteryl succinate modification of a glucomannan, named BSP, isolated from Bletilla striata tubers in order to make it amphiphilic and self-assemble into nanoparticles in aqueous solution. This study will expand the application scope of BSP in drug delivery for an efficient use of the traditional Chinese drug and will also provide a new idea for the exploration of natural product resources.

\section{Results and Discussion}

\subsection{Isolation and Purification of BSP}

The crude polysaccharide (yield 16.9\% of dried material) was obtained from the tubers of Bletilla striata via ultrasonic-assisted extraction, ethanol precipitation and Sevag reagent deproteination [10]. It was then purified using Sepharose CL-6B column chromatography to give the pure polysaccharide fraction BSP. BSP contained 96\% total carbohydrate as determined by the phenol-sulfuric acid method [10], but did not contain uronic acid as determined by the $m$-hydroxydiphenyl method [11]. BSP had no absorption at $260 \mathrm{~nm}$ and $280 \mathrm{~nm}$, showing it did not contain nucleic acid and protein. Sugar composition analysis revealed that BSP mainly consisted of mannose and glucose in the molar ratio of 3.5:1, consistent with the result that it contained no uronic acid. BSP exhibited a single and symmetrical peak both on GPC and HPGPC, showing that it was homogeneous. Its Mw of $20 \mathrm{kDa}$, as estimated by HPGPC using standard dextrans of known Mw, was lower than those of the polysaccharide fractions (135 kDa, $182 \mathrm{kDa}$ and $235 \mathrm{kDa}$ ) extracted by hot water [4,5,12]. This might be due to the ultrasonic-assisted extraction used in this study resulting in some degradation of the water soluble polysaccharides. 


\subsection{Structural Analysis of BSP}

The FT-IR spectrum of BSP (Figure 1) showed the characteristic absorption of $\beta$-type glycosidic linkages at $874 \mathrm{~cm}^{-1}$ [6]. The absorption band at $3443 \mathrm{~cm}^{-1}$ was assigned to hydroxyl stretching vibrations, the band at $2925 \mathrm{~cm}^{-1}$ to $\mathrm{C}-\mathrm{H}$ stretching vibrations, the band at $1743 \mathrm{~cm}^{-1}$ to the stretching vibration of the $\mathrm{C}=\mathrm{O}$ in the ester carbonyl groups, and the band at $1646 \mathrm{~cm}^{-1}$ to bound water [13]. The absorption at $1029 \mathrm{~cm}^{-1}$ was due to the pyranose configuration of the residues.

Figure 1. The FT-IR spectra of BSP and CHSB.

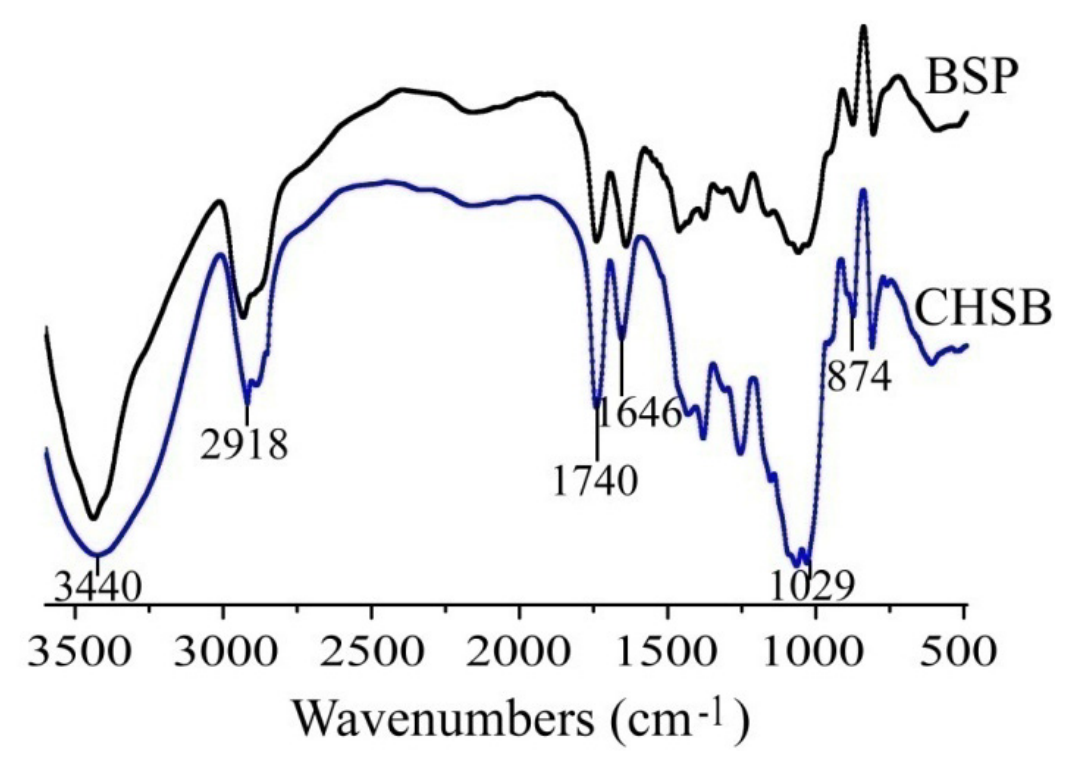

The fully methylated BSP was hydrolyzed with acid, converted into alditol acetates and analyzed by GC-MS. The results, summarized in Table 1, suggest that BSP was mainly composed of $(1 \rightarrow 4)$-linked-D-mannose and $(1 \rightarrow 4)$-linked-D-glucose. The molar ratio of the two glycosidic linkages was in agreement with that of the monosaccharide composition in BSP.

Table 1. Methylation analysis data for BSP.

\begin{tabular}{llll}
\hline Methylated Sugar & Linkages Types & Molar Ratio (\%) & Mass Fragment $(\boldsymbol{m} / \mathbf{z})$ \\
\hline 2, 3, 6-Me ${ }_{3}-\mathrm{Man} p$ & $\rightarrow$ 4)-D-Man- $(1 \rightarrow$ & 76.4 & $43,87,101,117$ \\
\hline & & & $129,161,203,233,277$ \\
\hline 2, 3, 6-Me $-\mathrm{Glc} p$ & $\rightarrow$ 4)-D-Glc-(1 $\rightarrow$ & 20.3 & $43,87,99,101$ \\
\hline & & & $117,129,189,233$ \\
\hline
\end{tabular}

Further structural features of BSP were determined by ${ }^{13} \mathrm{C}$ - and ${ }^{1} \mathrm{H}-\mathrm{NMR}$ spectroscopy (Figure 2). Assignments of signals and identification of the sugar residues were done by combinations of two-dimensional HSQC analysis (Figure 3) and comparison of the chemical shifts with published data [14-16]. All the results are summarized in Table 2. In the ${ }^{13} \mathrm{C}-\mathrm{NMR}$ spectrum, the signal at $30.4 \mathrm{ppm}$ was due to the methyl carbon in the acetone used as the internal standard reference. The anomeric signal at 100.26 ppm was attributed to $\mathrm{C}-1$ of 1,4-linked $\beta$-Man. Other signals at 70.09, 71.57, 76.64, 75.15 and 60.59 ppm were attributed to C-2, C-3, C-4, C-5 and C-6 of 1,4-linked $\beta$-Man, respectively. The anomeric signal at $102.07 \mathrm{ppm}$ was assigned to 1,4 -linked $\beta$-Glc, and the signal at $78.55 \mathrm{ppm}$ 
belonged to the C-4 of 1,4-linked $\beta$-Glc. The signal at $173.5 \mathrm{ppm}$ and $20.7 \mathrm{ppm}$ were assigned to carbonyl carbon and methyl carbon in acetyl groups, respectively. This was consistent with the FT-IR result and suggested the presence of acetyl groups in BSP.

In the ${ }^{1} \mathrm{H}-\mathrm{NMR}$ spectrum, signals at 4.70, 4.06, 3.72, 3.75, 3.50, 3.89/3.69 ppm were related to $\mathrm{H}-1$ to H-6 of 1,4-linked $\beta$-Man, respectively. The signal of 4.46 ppm was attributed to H-1 of 1,4-linked $\beta$-Glc. The highest-field signal, at $2.10 \mathrm{ppm}$, belonged to the methyl of an acetyl group. In the low field, there was a signal at $5.41 \mathrm{ppm}$, which might be assigned to $\mathrm{H}-2$ of 2-O-acetyl-1,4-linked $\beta$-Man [17].

According to the methylation analysis, FT-IR and NMR results, BSP was thus determined to be an acetylglucomannan having a backbone of 1,4-linked $\beta$-D-Man and $\beta$-D-Glc in a ratio of $~ 3.5: 1$. This structure was different from the glucomannan $(\mathrm{BSPb})$ fraction isolated from Bletilla striata, which was composed of 1,2-linked $\alpha$-D-Man and 1,4-linked $\beta$-D-Glc with a ratio of 3:1 [6], but was similar to the polysaccharide "Bletilla-glucomannan" with both 1,4-linked $\beta$-D-Man and $\beta$-D-Glc in a ratio of 3:1 [4], and the polysaccharide BSPF2 with 1,4-linked $\beta$-D-Man and $\beta$-D-Glc in a ratio of 2:1, although three fifths of the Glc residues in BSPF2 also substituted by terminal Man residues [5]. The structural differences between BSP and other Bletilla striata polysaccharides might be caused by the different extraction methods. A polysaccharide with similar structure was found in the dried stem of Dendrobium officinale, which had a backbone of 1,4-linked $\beta$-D-Man and $\beta$-D-Glc, with branches at O-6 consisting of terminal and 1,3-linked Man, 1,3-linked Glc, and a small proportion of arabinofuranosyl residues at the terminal position [17]. An acetylated glucomannan was also obtained from Cyrtopodium andersonii, which had a backbone of 1,4-linked $\beta$-D-Man and $\beta$-D-Glc and substituted by acetyl groups at C-2 of the 1,4-linked $\beta$-D-Man units [18].

Figure 2. (A) ${ }^{13} \mathrm{C}-\mathrm{NMR}$ spectrum of BSP; (B) ${ }^{1} \mathrm{H}-\mathrm{NMR}$ spectrum of BSP.
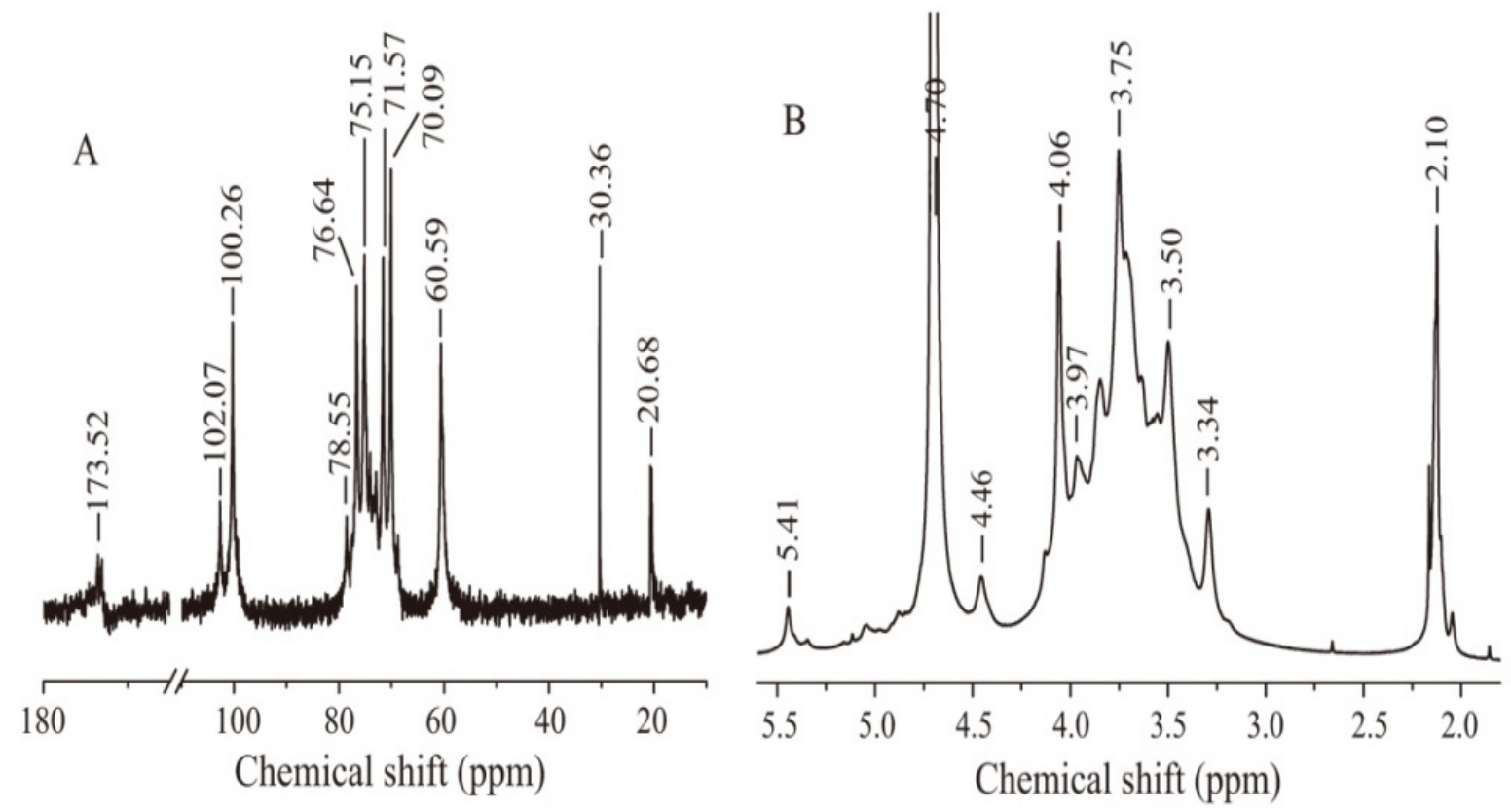
Figure 3. HSQC NMR spectrum of BSP.

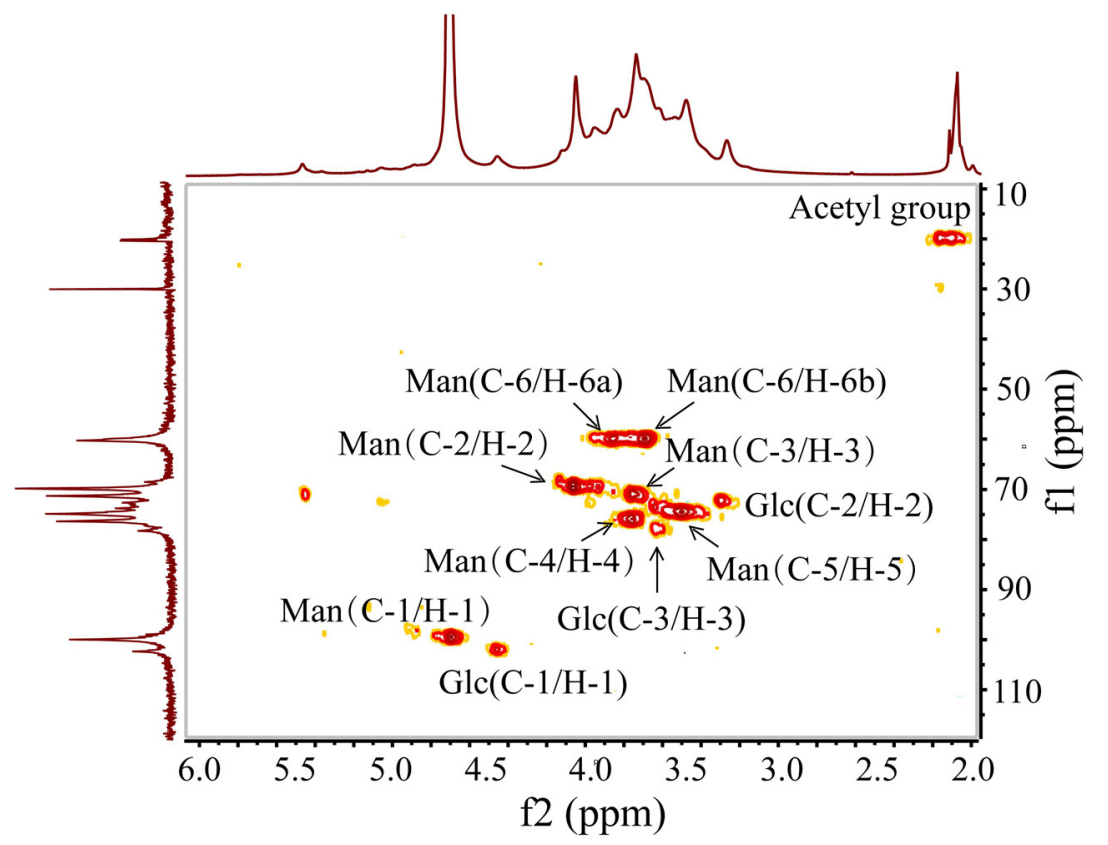

Table 2. ${ }^{13} \mathrm{C}-\mathrm{NMR}$ and ${ }^{1} \mathrm{H}-\mathrm{NMR}$ spectral assignments of BSP.

\begin{tabular}{|c|c|c|c|c|c|c|}
\hline \multirow{2}{*}{ Sugar Residues } & \multicolumn{5}{|c|}{ Chemical Shifts ${ }^{\mathbf{1 3}} \mathbf{C} /{ }^{\mathbf{1}} \mathbf{H} \mathbf{\delta}(\mathbf{p p m})$} \\
\cline { 2 - 8 } & $\mathbf{1}$ & $\mathbf{2}$ & $\mathbf{3}$ & $\mathbf{4}$ & $\mathbf{5}$ & $\mathbf{6}$ \\
\hline \multirow{2}{*}{$\rightarrow 4)-\beta$-D-Man- $(1 \rightarrow$} & 100.26 & 70.09 & 71.57 & 76.64 & 75.15 & 60.59 \\
\cline { 2 - 8 } & 4.70 & 4.06 & 3.72 & 3.75 & 3.50 & $3.89 / 3.69$ \\
\hline \multirow{2}{*}{$\rightarrow 4)-\beta$-D-Glc- $(1 \rightarrow$} & 102.07 & 72.17 & 75.89 & 78.55 & 74.5 & 60.69 \\
\cline { 2 - 7 } & 4.46 & 3.34 & 3.76 & 3.64 & 3.58 & $3.77 / 3.65$ \\
\hline
\end{tabular}

\subsection{Determination of Degree of Substitution (DS) for Cholesteryl Succinate Modified BSP (CHSB)}

In order to increase the hydrophobic domain of BSP, it was modified by reaction with cholesteryl succinate (CHS) catalyzed by 1-ethyl-(3-dimethylaminopropyl) carbonylimine hydrochloride (EDC) and triethylamine. After CHS was connected to the BSP molecule, a hydrophobic modified BSP (CHSB) was obtained. The FT-IR spectra of BSP and CHSB are shown in Figure 1. Compared with the spectrum of BSP, the absorption at about $1740 \mathrm{~cm}^{-1}$, which was assigned to the stretching vibrations of the carbonyl group, was remarkably increased in CHSB with the substitution by CHS. At the same time, the increase of the methylene stretching vibration at about $2920 \mathrm{~cm}^{-1}$ was observed. These results indicated that the modification of BSP by CHS proceeded successfully.

It is reported that sulfuric acid could hydrolyze deoxycholate which was covalently bound to dextran-modified polysaccharides [19]. By detecting the amount of deoxycholate by UV spectrophotometry, the degree of substitution by deoxycholate in the modified polysaccharide could be obtained. Inspired by this method, ammonia ferric sulfate was used in this study to hydrolyze CHSB and the degree of substitution by CHS in CHSB was determined by a colorimetric method [20]. Using Equation (1), the degree of substitution for CHSB was determined to be $3.2 \%$ :

$$
\mathrm{DS}=\left(\mathrm{C} / \mathrm{M}_{1}\right) /\left((\mathrm{m}-\mathrm{C}) / \mathrm{M}_{2}\right)
$$


where, $\mathrm{M}_{1}$ is the molar mass of cholesterol $387 ; \mathrm{M}_{2}$ is the molar mass of a sugar unit structure 162; $\mathrm{C}$ is for the concentration of cholesterol and $\mathrm{m}$ is the weight of CHSB $15 \mathrm{mg}$.

\subsection{Determination of the Critical Micelle Concentration (CMC) of CHSB by Pyrene Fluorescence} Probe Spectrometry

Pyrene is used as a hydrophobic fluorescent probe [21] due to its low solubility, self-quenching effect and relatively low fluorescence intensity in polar environment. Therefore, when a polar solvent (water) exists in a micellar or hydrophobic domain, pyrene will spontaneously metastasize to the nonpolar environment, leading to significant increase of fluorescence intensity. In aqueous solution, CHSB could form a hydrophobic domain, therefore pyrene would shift into this hydrophobic domain, producing changes in the emission spectrum accordingly. By measuring the fluorescence intensity at $\mathrm{I}_{372}$ and $\mathrm{I}_{383}$, the CMC of CHSB could be determined.

Figure 4 showed the fluorescence excitation ratio of $\mathrm{I}_{372} / \mathrm{I}_{383}$ for CHSB and BSP in correlation with $\log$ concentration. The concentration of BSP had nothing to do with the ratio of $\mathrm{I}_{372} / \mathrm{I}_{383}$. When the concentration of CHSB was low, the ratio of $\mathrm{I}_{372} / \mathrm{I}_{383}$ basically remained unchanged. When the concentration exceeded the critical concentration, the ratio of $\mathrm{I}_{372} / \mathrm{I}_{383}$ increased dramatically along with the increase of concentration. Aguiar et al. [22] reported that the relationship between the fluorescence excitation ratio $\mathrm{I}_{372} / \mathrm{I}_{383}$ and the concentration was in accordance with the Boltzamann curve rule:

$$
\mathrm{y}=\left[\left(\mathrm{A}_{1}-\mathrm{A}_{2}\right) /\left(1+\mathrm{e}^{\left.\left(\mathrm{x}-\mathrm{x}_{0}\right) / \Delta \mathrm{x}\right)}\right]+\mathrm{A}_{2}\right.
$$

Figure 4. The diagram of the fluorescence excitation spectra intensity ratio $I_{372} / I_{383}$ and the logarithmic (Log) concentration of CHSB and BSP. BSP was used as a control.

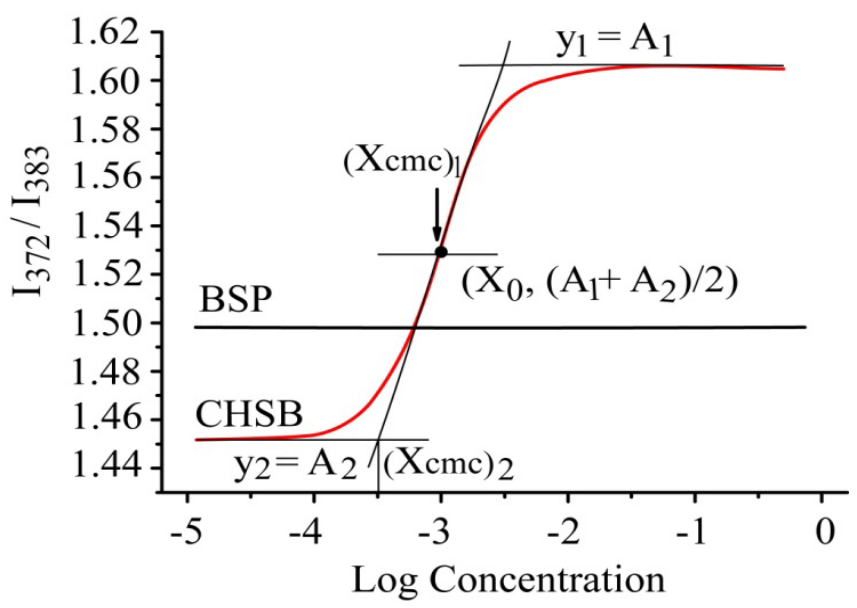

In Equation (2), $\mathrm{x}_{0}$ is the center of the sigmoid and $\mathrm{x}$ is directly related to the independent variable range where the abrupt change of the dependent variable occurs. $A_{1}$ is the ratio of $I_{372} / I_{383}$ under surfactant with low concentration and $A_{2}$ is the ratio of $I_{372} / I_{383}$ under surfactant with high concentration. When $\mathrm{x}_{0} / \Delta \mathrm{x}<10$ (where $\left.\Delta \mathrm{x}=\left(\left(\mathrm{x}_{\mathrm{CMC}}\right)_{2}-\mathrm{x}_{0}\right) / 2\right), \mathrm{CMC}=\left(\mathrm{x}_{\mathrm{CMC}}\right)_{1}$; otherwise, $\mathrm{CMC}=\left(\mathrm{x}_{\mathrm{CMC}}\right)_{2}$. After nonlinear curve fitting, the following results were obtained: $\left(\mathrm{x}_{\mathrm{CMC}}\right)_{1}=0.001 \mathrm{mg} / \mathrm{mL}$ $\left(\mathrm{x}_{\mathrm{CMC}}\right)_{2}=0.483 \mathrm{mg} / \mathrm{mL}, \mathrm{x}_{0}=9.6 \times 10^{-4} \mathrm{mg} / \mathrm{mL}$. Therefore, $\mathrm{x}_{0} / \Delta \mathrm{x}=0.004<10$. According to what is mentioned above, $\mathrm{CMC}=\left(\mathrm{x}_{\mathrm{CMC}}\right)_{1}$, which meant the intersection point corresponded to the CMC. This 
result showed that the CMC of CHSB was $0.001 \mathrm{mg} / \mathrm{mL}$, less than that of many other small molecule surfactants, for example, the CMC of sodium dodecyl sulfate was $2.3 \mathrm{mg} / \mathrm{mL}$ [23] and the CMC of deoxycholate was $1.0 \mathrm{mg} / \mathrm{mL}$ [24]. Therefore, CHSB was a novel kind of amphiphilic macromolecule material and could self-assemble into nanoparticles in aqueous solution. This low CMC of CHSB shows that it would have a good stability in physiological liquid environment and is expected to be useful in drug delivery systems. Cholesteryl groups are hydrophobic segments which possess good biocompatibility which is a great ability to drive self-assembly, as well as strong potential interactions with cholesteryl receptors on the cell surface. Cholesteryl-modified hydroxypropyl cellulose had been well studied $[25,26]$. These cellulose derivatives had good loading capacity for hydrophobic drugs and controlled-release properties [27]. Therefore, we hypothesize that CHSB would be a novel nanocarrier for hydrophobic drug delivery.

\section{Experimental Section}

\subsection{Materials}

The tubers of Bletilla striata were bought from Nanjing Institute of Comprehensive Utilization of Wild Plant. Sepharose CL-6B gel was obtained from Amersham Pharmacia Biotech (Uppsala, Sweden). All other reagents used were of analytical grade made in China.

\subsection{General Methods}

The total sugar content was determined by a phenol-sulfuric acid assay using Glc as standard [28]. Sepharose CL-6B chromatography was performed on a $1.5 \times 90 \mathrm{~cm}$ column, eluating with $0.15 \mathrm{M}$ $\mathrm{NaCl}$ at a flow rate of $0.15 \mathrm{~mL} / \mathrm{min}$. Dialysis was carried out using tubing with Mw cut-off $3500 \mathrm{Da}$ (for globular proteins). FT-IR spectra were obtained in a range of $4000-400 \mathrm{~cm}^{-1}$ on a Nicolet 6700 FT-IR spectrometer (ThermoScientific, Waltham, MA, USA) with a DTGS detector. The sample was measured as a film on $\mathrm{KBr}$ discs. The NMR experiments were conducted as described by Zhang [10]. BSP (20 mg) was dissolved in $\mathrm{D}_{2} \mathrm{O}(99.8 \%, 0.5 \mathrm{~mL})$, freeze-dried, re-dissolved in $\mathrm{D}_{2} \mathrm{O}(0.5 \mathrm{~mL})$, and centrifuged to remove the excess sample.

\subsection{Isolation and Purification}

Bletilla striata tuber powder ( $20 \mathrm{~g}$ ) was immersed in 20 volumes of distilled water and submitted to ultrasonic-assisted extraction with water at $80{ }^{\circ} \mathrm{C}$ for $10 \mathrm{~min}$. The aqueous filtrates were combined and concentrated to one-tenth of the original volume. To precipitate the polysaccharides, $95 \%$ ethanol was added to the aqueous filtrates to reach $80 \%$ of the final volume. The polysaccharides were collected by centrifugation and dried in vacuum. The supernatant was treated with Sevag reagent (1:4 n-butanol/chloroform, $\mathrm{v} / \mathrm{v})$ to remove the free proteins [10]. The deproteinated polysaccharide $(5 \mathrm{~g})$ was dissolved in distilled water $(10 \mathrm{mg} / \mathrm{mL})$ and centrifuged. The supernatant was applied on a Sepharose CL-6B column $(3.0 \times 90 \mathrm{~cm})$ and eluted with $0.15 \mathrm{M} \mathrm{NaCl}$ solution at a flow rate of $0.5 \mathrm{~mL} / \mathrm{min}$. The eluate was collected $10 \mathrm{~mL}$ per tube. The main peak was collected according to the sugar profile detected by phenol-sulfuric acid method, concentrated, dialyzed and lyophilized to yield BSP. 


\subsection{Homogeneity and Molecular Weight}

Homogeneity and molecular weight were determined by HPGPC performed on a Shimadzu, 10Avp linked gel filtration column of TSK-G4000 $\mathrm{PW}_{\mathrm{XL}}$, eluting with $0.2 \mathrm{M} \mathrm{NaCl}$ at $0.5 \mathrm{~mL} / \mathrm{min}, 35.0 \pm 0.1{ }^{\circ} \mathrm{C}$, monitored using a refractive index RID-10A detector (Shimadzu, Kyoto, Japan). A sample solution $20 \mu \mathrm{L}$ $(5 \mathrm{mg} / \mathrm{mL})$ was injected in the column. The gel filtration column was calibrated by standard dextrans (410 kDa, $150 \mathrm{kDa}, 50 \mathrm{kDa}, 5 \mathrm{kDa}$ ) using linear regression.

\subsection{Monosaccharide Composition}

Monosaccharide analysis was performed by a HPLC method as described by Zhang [10]. The polysaccharide BSP $(2 \mathrm{mg}$ ) was hydrolyzed using $1.0 \mathrm{~mL}$ anhydrous methanol containing $2 \mathrm{M}$ $\mathrm{HCl}$ at $80{ }^{\circ} \mathrm{C}$ for $16 \mathrm{~h}$ and then with $1.0 \mathrm{~mL}$ of $2 \mathrm{M} \mathrm{CF}_{3} \mathrm{COOH}(0.5 \mathrm{~mL})$ at $120{ }^{\circ} \mathrm{C}$ for $1 \mathrm{~h}$. The hydrolysis-product was derivatized with $0.5 \mathrm{M}$ 1-phenyl-3-methyl-5-pyrazolone derivatives and $0.3 \mathrm{M} \mathrm{NaOH}$. After neutralization with $0.3 \mathrm{M} \mathrm{HCl}$, the derivatives were analyzed by HPLC on a DIKMA Inertsil ODS-3 column $(150 \times 4.6 \mathrm{~mm}$ i.d. $)$ with a guard column on a Shimadzu HPLC system (LC-10ATvp pump and UV-VIS detector) and monitored by UV absorbance at $245 \mathrm{~nm}$.

\subsection{Methylation Analysis}

BSP was methylated using the Ciucanu and Kerek method [29]. BSP (20 mg) was dissolved in DMSO $(2 \mathrm{~mL})$ and methylated by treatment with $\mathrm{NaOH}-\mathrm{DMSO}(2 \mathrm{~mL})$ suspension and iodomethane $(1.8 \mathrm{~mL})$. The reaction mixture was extracted with $\mathrm{CHCl}_{3}$, and then the solvent was removed by vacuum evaporation. Complete methylation was confirmed by the disappearance of the -OH band (3200-3700 $\mathrm{cm}^{-1}$ ) in the FT-IR spectrum. The permethylated product was hydrolyzed subsequently by $\mathrm{HCOOH}(85 \%, 0.5 \mathrm{~mL})$ for $4 \mathrm{~h}$ at $100{ }^{\circ} \mathrm{C}$, and then by $\mathrm{CF}_{3} \mathrm{COOH}(2 \mathrm{M}, 1 \mathrm{~mL})$ for $6 \mathrm{~h}$ at $100{ }^{\circ} \mathrm{C}$. The mixture was evaporated to dryness, followed by reduction with $\mathrm{NaBH}_{4}$ and acetylation with acetic anhydride. The resulting mixture of the methylated alditol acetates were analyzed by GC-MS using a Shimadzu GC-14C instrument.

\subsection{Synthesis of Cholesteryl Succinate}

Cholesterol (25 g) and succinic anhydride (20 g) were dissolved in anhydrous pyridine $(20 \mathrm{~mL})$ and maintained at room temperature for $48 \mathrm{~h}$. The $\mathrm{pH}$ was adjusted to 1-2 with hydrochloric acid solution under ice cooling (hydrochloric acid/ice/water $=12 / 50 / 40$ ). The mixture was kept at $4{ }^{\circ} \mathrm{C}$ overnight. After filtration, the precipitate was collected and washed with distilled water until $\mathrm{pH}>5$. The precipitate was recrystallized in ethyl acetate/ethanol system and dried at $80{ }^{\circ} \mathrm{C}$ to obtain a pure white needle-like cholesteryl succinate product.

\subsection{Synthesis Cholesteryl Succinate - BSP (CHSB)}

BSP polysaccharide $(0.5 \mathrm{~g})$ was dissolved in dimethyl formamide (DMF, $15 \mathrm{~mL})$. CHS, EDC and triethylamine $(\mathrm{EDC} / \mathrm{CHS} /$ triethylamine $=1.2 / 1 / 1)$ were dissolved in DMF $(10 \mathrm{~mL})$ and stirred at room temperature for $1 \mathrm{~h}$. The above solution was added to the BSP polysaccharide solution and kept 
for $48 \mathrm{~h}$. Anhydrous ethanol $(200 \mathrm{~mL})$ was added to the reaction mixture and a white precipitate was obtained. The resulting precipitate was separated by centrifugation, washed subsequently with ethanol, tetrahydrofuran and ether and finally dried at $80{ }^{\circ} \mathrm{C}$.

\subsection{Determination of the Degree of Substitution of CHSB}

CHSB $(7.5 \mathrm{mg})$ was mixed with $50 \% \mathrm{KOH}$ solution $(4.0 \mathrm{~mL})$ and anhydrous ethanol $(2.0 \mathrm{~mL})$ in a $25 \mathrm{~mL}$ colorimetric tube. The mixture was submitted to ultrasonic-assisted dissolution for $5 \mathrm{~min}$ and saponification for $2 \mathrm{~h}$ at $65{ }^{\circ} \mathrm{C}$ on a water bath under shaking. After complete saponification, $3 \mathrm{~mL} \mathrm{5 \%}$ $\mathrm{NaCl}$ solution was added to the mixed solution and cooled at room temperature. After addition of petroleum ether $(10 \mathrm{~mL})$ with vigorous stirring for $1 \mathrm{~min}$, it was left for stratification. One $\mathrm{mL}$ of the upper petroleum ether solution was drawn into a stoppered colorimetric tube and was dried under natural evaporation at $65^{\circ} \mathrm{C}$ water bath. Ethanol $(1 \mathrm{~mL})$ was added and jiggled to make the cholesterol dissolve. The degree of substitution for CHSB was calculated from the change of absorption at $560 \mathrm{~nm}$ using the standard curve of cholesterol and the formula DS $=\left(C / M_{1}\right) /\left((m-C) / M_{2}\right)$, where $M_{1}$ is the molar mass of cholesterol 387; $\mathrm{M}_{2}$ is the molar mass of a sugar unit structure 162 ; $\mathrm{C}$ is for the concentration of cholesterol and $\mathrm{m}$ is the weight of CHSB $15 \mathrm{mg}$.

\subsection{Determination of CMC of CHSB}

For steady-state pyrene fluorescence probe spectrometry, the CHSB sample was dissolved in 2\% acetic acid solution $(1 \mathrm{mg} / \mathrm{mL})$ and dialyzed against distilled water for $24 \mathrm{~h}$. The non-dialyzed portion was filtered with a microporous membrane and the filtrate was submitted to ultrasonic-assisted treatment at $37{ }^{\circ} \mathrm{C}$ [30] followed by addition of methanol-pyrene solution in a $10 \mathrm{~mL}$ flask. The concentration of pyrene was adjusted to $3 \times 10^{-6} \mathrm{~mol} / \mathrm{L}$ after adding $\mathrm{CHSB}$. The solution was submitted to ultrasonic-assisted treatment for $40 \mathrm{~min}$ and put on $37{ }^{\circ} \mathrm{C}$ water bath for $1 \mathrm{~h}$ under oscillation. After scanning at wavenumbers from $350 \mathrm{~nm}$ to $450 \mathrm{~nm}$, the fluorescence emission spectrum was obtained and the excitation wavelength was set at $335 \mathrm{~nm}$. By recording the fluorescence intensity at the absorption of $372 \mathrm{~nm}$ and $383 \mathrm{~nm}$ [31], the ratio of $\mathrm{I}_{372} / \mathrm{I}_{383}$ to the numerical $\log$ concentration was plotted to obtain CMC of CHSB, which corresponded to the middle point of curve inflexion.

\section{Conclusions}

From the results obtained in our study, we could conclude that the polysaccharide BSP extracted from Bletilla striata was a glucomannan with a Mw of $20 \mathrm{kDa}$. Methylation analysis, FT-IR and NMR spectra revealed that BSP was an acetylglucomannan having a backbone of 1,4-linked $\beta$-D-Man and $\beta$-D-Glc in a ratio of $\sim 3.5: 1$. A chemical method for the modification of glucomannan by cholesteryl succinate was also established in this study and a modified product CHSB was obtained. The degree of substitution by cholesteryl succinate in CHSB was 3.2\%. Using the steady-state fluorescence probe method, the critical micelle concentration of CHSB was determined to be $0.001 \mathrm{mg} / \mathrm{mL}$, less than that of many other small molecule surfactants, which indicated CHSB was a novel kind of amphiphilic macromolecule material and could self-assemble into nanoparticles in aqueous solution. This study 
provided data and information that should help expand the utilization of BSP in drug delivery studies, expand the traditional Chinese drug industry and provide a novel concept for the exploration of natural product resources.

\section{Acknowledgments}

This work was supported by the National Natural Science Foundation of China (No. 81173559), the Key Scientific Program of Jilin Province (No. YYZX201128), and Jilin Province Key Laboratory on Chemistry and Biology of Natural Drugs in Changbai Mountain.

\section{Author Contributions}

Mengshan Zhang was responsible for data acquisition, analysis and manuscript preparation. Lin Sun and Xiaoxia Peng helped to analyze the data. Wencui Zhao and Fuqiang Liu worked on the preparation of the studied materials. Yajing Bi, Yanping Wang and Hengbi Zhang were responsible for the experimental design. Yifa Zhou was responsible for the programs and all experiments, critically revised the manuscript and provided final approval for the article.

\section{Conflicts of Interest}

The authors declare no conflict of interest.

\section{References}

1. Sun, D.F.; Shi, J.S.; Zhang, W.M.; Gu, G.P.; Zhu, C.L.; Xue, H.M. Research Progress on Polysaccharide Gum of Bletilla striata (Thunb.) Reichb.f. Food Sci. 2009, 30, 296-298.

2. Wu, X.G.; Xin, M.; Chen, H.; Yang, L.N.; Jiang, H.R. Novel mucoadhesive polysaccharide isolated from Bletilla striata improves the intraocular penetration and efficacy of levofloxacin in the topical treatment of experimental bacterial keratitis. J. Pharm. Pharmacol. 2010, 62, 1152-1157.

3. Li, W.Y.; Zhan, H.; Fang, K.; Chen, H.T.; Zheng, C.S. Cisplatin Bletilla striata gel microspheres in dogs in vivo pharmacokinetics. Chin. J. Hosp. Pharm. 2008, 28, 524-527.

4. Tomoda, M.; Nakatsuka, S.; Tamai, M.; Nagata, M. Plant mucilages. VIII. Isolation and characterization of a mucous polysaccharide, Bletilla-glucomannan, from Bletilla striata tubers. Chem. Pharm. Bull. 1973, 21, 2667-2671.

5. Peng, Q.; Li, M.; Xue, F.; Liu, H.J. Structure and immunobiological activity of a new polysaccharide from Bletilla striata. Carbohydr. Polym. 2014, 107, 119-123.

6. Wang, Y.; Liu, D.; Chen, S.J.; Wang, Y.; Jiang, H.X.; Yin, H.P. A new glucomannan from Bletilla striata: Structural and anti-fibrosis effects. Fitoterapia 2014, 92, 72-78.

7. Diao, H.J.; Li, X.; Chen, J.N.; Luo, Y.; Chen, X.; Dong, L.; Wang, C.M.; Zhang, C.Y.; Zhang, J.F. Bletilla striata polysaccharide stimulates inducible nitric oxide synthase and proinflammatory cytokine expression in macrophages. J. Biosci. Bioeng. 2008, 2, 85-89. 
8. Akiyama, E.; Morimoto, N.; Kujawa, P.; Ozawa, Y.; Winnik, F.M.; Akiyoshi, K. Self-assembled nanogels of cholesteryl-modified polysaccharides: Effect of the polysaccharide structure on their association characteristics in the dilute and semi dilute regimes. Biomacromolecules 2007, 8 , 2366-2373.

9. Yang, L.; Kuang, J.; Li, Z.; Zhang, B.; Cai, X.; Zhang, L.M. Amphiphilic cholesteryl-bearing carboxymethyl-cellulose derivatives: Self-assembled and rheological behavior in aqueous solution. Cellulose 2008, 15, 659-669.

10. Zhang, X.; Yu, L.; Bi, H.T.; Li, X.H.; Ni, W.H.; Han, H.; Li, N.; Wang, B.Q.; Zhou, Y.F.; Tai, G.H. Total fractionation and characterization of the water-soluble polysaccharides isolated from Panax ginseng C. A. Meyer. Carbohydr. Polym. 2009, 77, 544-552.

11. Blumenkrantz, N.; Asboe-Hansen, G. New method for quantitative determination of uronic acids. Anal. Biochem. 1973, 54, 484-489.

12. Wang, C.M.; Sun, J.T.; Luo, Y.; Xue, W.H.; Diao, H.J.; Dong, L.; Chen J.N.; Zhang, J.F. A polysaccharide isolated from the medicinal herb Bletilla striata induces endothelial cells proliferation and vascular endothelial growth factor expression in vitro. Biotechnol. Lett. 2006, $28,539-543$.

13. Li, M.F.; Sun, S.N.; Xu, F.; Sun, R.C. Ultrasound-enhanced extraction of lignin from bamboo (Neosinocalamu saffinis): Characterization of the ethanol-soluble fractions. Ultrason. Sonochem. 2012, 19, 243-249.

14. Aguire, M.J.; Isaacs, M.; Matsuhiro, B.; Mendoza, L.; Zunigai, E.A. Characterization of a neutral polysaccharide with antioxidant capacity from red wine. Carbohydr. Res. 2009, 344, 1095-1101.

15. Chakraborty, I.; Mondal, S.; Pramanik, M.; Rout, D.; Islam, S.S. Structural investigation of a water-soluble glucan from an edible mushroom, Astraeus hygrometricus. Carbohydr. Res. 2004, 339, 2249-2254.

16. Capek, P. A water soluble glucomannan isolated from an immunomodulatory activepolysaccharide of Salvia officinalis L. Carbohydr. Polym. 2009, 75, 356-359.

17. Hua, Y.F.; Zhang, M.; Fu, C.X.; Chen, Z.H.; Chan, G.Y.S. Structural characterization of a 2-O-acetylglucomannan from Dendrobium officinale stem. Carbohydr. Res. 2004, 339, 2219-2224.

18. Parente, J.P.; Adão, C.R.; Silva, B.P.D.; Tinoco, L.W. Structural characterization of an acetylated glucomannan with antiinflammatory activity and gastroprotective property from Cyrtopodium andersonii. Carbohydr. Res. 2014, 391, 16-21.

19. Yuan, X.B.; Li, H.; Zhu, X.X.; Woo, H.G. Self-aggregated nanoparticles composed of periodate-oxidized dextran and cholic acid: Preparation, stabilization and in vitro drug release. J. Chem. Technol. Biotechnol. 2006, 81, 746-54.

20. Ding, Z.P.; Wang, M.H.; Liu, Z.H.; He, Y.; Zhao, D.Y. The determination of the cholesterol concentration in the food product and the study of the method. Food Sci. 2004, 25, 130-135.

21. Zhao, Z.; Wang, Q.F. Progress on methods of measuring surface active agents critical micelle concentration. Pract. Pharm. Clin. Rem. 2010, 13, 140-144.

22. Aguiar, J.; Carpena, P.; Molina-Bolívar, J.A.; Ruiz, C.C. On the determination of the critical micelle concent ration by the pyrene 1:3 ratio method. J. Colloid. Interface Sci. 2003, 258, 116-122.

23. Rahman, A.; Brown, C.W. Effect of $\mathrm{pH}$ on the critical micelle concentration of sodium dodecyl sulphate. J. Appl. Polym. Sci. 2003, 28, 1331-1334. 
24. Kratohvil, J.P.; Hsu, W.P.; Kwok, V. How large are the micelles of di- $\alpha$-hydroxy bile salts at the critical micellization concentrations in aqueous electrolyte solutions? Results for sodium taurodeoxycholate and sodium deoxycholate. Langmuir 1986, 2, 256-258.

25. Bagheri, M.; Pourmirzaei, L. Synthesis and characterization of cholesteryl-modified graft copolymer from hydroxypropyl cellulose and its application as nanocarrier. Macromol. Res. 2013, 21, 801-808.

26. Bagheri, M.; Shateri, S. Synthesis and characterization of novel liquid crystalline cholesteryl-modified hydroxypropyl cellulose derivatives. J. Polym. Res. 2012, 19, 9842.

27. Bagheri, M.; Shateri, S. Thermosensitive nanosized micelles from cholesteryl-modified hydroxypropyl cellulose as a novel carrier of hydrophobic drugs. Iran. Polym. J. 2012, 21, 365-373.

28. Dubois, M.; Gilles, K.A.; Hamilton, J.K.; Rebers, P.A.; Smith, F. Colorimetric method for determination of sugars and related substances. Anal. Chem. 1956, 28, 350-356.

29. Ciukanu, I.; Kerek, F. A simple and rapid method for the permethylation of carbohydrates. Carbohydr. Res. 1984, 131, 209-217.

30. Wang, Y.S. Preparation of Self-Aggregated Nanopartieles of Cholesterol-Bearing Chitosan Derivatives and the Primary Study on Using Them as the Novel Carriers of drUgs. Ph.D. Thesis, Chinese Academy of Medical Sciences \& Peking Union Medical College, Beijing, China, May 2009.

31. Lu, G.Q.; Zheng, J.; Zheng, X.C.; Lu, Y.L.; He, F.C. The study of aggregation behavior of bile salt-phosphatidylcholine mixed nano-micelle by pyrene fluorescence probe spectrometry methods and curve fitting. Drug Res. 2011, 20, 21-23.

Sample Availability: Samples of the compounds are available from the authors.

(C) 2014 by the authors; licensee MDPI, Basel, Switzerland. This article is an open access article distributed under the terms and conditions of the Creative Commons Attribution license (http://creativecommons.org/licenses/by/3.0/). 